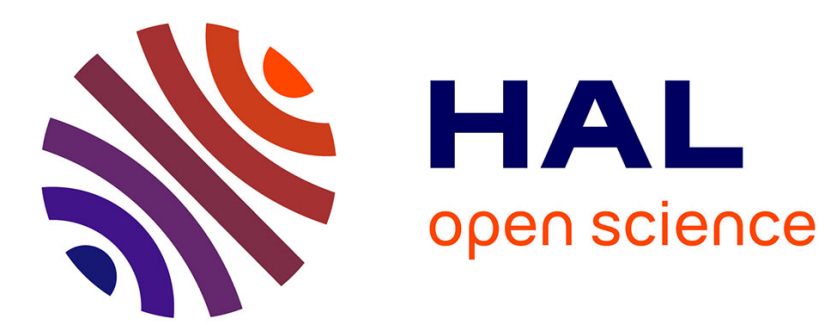

\title{
Internal Structuring of Silicon using THz-Repetition-Rate Trains of Ultrashort Pulses
}

\author{
A. Wang, A. Das, D. Grojo
}

\section{To cite this version:}

A. Wang, A. Das, D. Grojo. Internal Structuring of Silicon using THz-Repetition-Rate Trains of Ultrashort Pulses. OSA Technical Digest (Optical Society of America), 2020, pp.ATu3K.4. 10.1364/CLEO_AT.2020.ATu3K.4. hal-02990021

\section{HAL Id: hal-02990021 \\ https://hal.science/hal-02990021}

Submitted on 5 Nov 2020

HAL is a multi-disciplinary open access archive for the deposit and dissemination of scientific research documents, whether they are published or not. The documents may come from teaching and research institutions in France or abroad, or from public or private research centers.
L'archive ouverte pluridisciplinaire HAL, est destinée au dépôt et à la diffusion de documents scientifiques de niveau recherche, publiés ou non, émanant des établissements d'enseignement et de recherche français ou étrangers, des laboratoires publics ou privés. 


\title{
Internal Structuring of Silicon using THz-Repetition-Rate Trains of Ultrashort Pulses
}

\author{
A. Wang, A. Das, D. Grojo \\ Aix Marseille Université, CNRS, LP3, UMR7341, 13288 Marseille, France \\ Author e-mail address: andong.wang @univ-amu.fr,david.grojo@univ-amu.fr
}

\begin{abstract}
We generate and apply trains of infrared femtosecond pulses at the highest achievable repetition-rates. This gives unique multi-timescale control parameters used for improved energy deposition and reliable 3D laser writing deep inside silicon. (O2020 The Author(s)
\end{abstract}

\section{Introduction}

Ultrafast laser 3D inscription inside materials is today used in some of the most advanced technologies from 3D quantum photonics and optical communication to nanosurgery [1,2]. While this would be highly beneficial for the semiconductor industry, they however remain so far limited to transparent materials including dielectrics and some polymers. We have recently established that narrow bandgap semiconductors make hardly achievable local material manipulation in the bulk because of strong propagation nonlinearities experienced by ultrafast infrared light in these materials [3-5]. Although many progresses have been made recently, they are still inaccessible with the shortest pulses unless complex schemes are implemented. For example, we have demonstrated the first bulk modification inside silicon with femtosecond laser pulses by applying a solid-immersion focusing strategy [4]. However, the contact nature of the technique creates severe difficulties for implementations. In this manuscript, we describe a new solution which holds promises for application because of a possibility for implementation with a simple arrangement of crystals in the beam path.

\section{Methods}

The solution proposed in this paper relies on a multi-time scale optimization of the interactions. It is inspired by the new ultrafast GHz-burst mode of irradiation that has been recently introduced for unprecedented performances in femtosecond laser surface machining [6]. However, ours require pulse trains at even higher repetition rates so that accumulation effects can give their full potential before dissipation processes (including carrier and temperature diffusions [7]) come into play in silicon. We use a femtosecond laser system (Pharos) to pump an optical parameter amplifier delivering pulses of duration <200fs at $1550-\mathrm{nm}$ wavelength. The output energy was controlled by the combination of a half-waveplate and polarizer. A parallel grating pairs is built to stretch the pulses up to $21 \mathrm{ps.}$

To generate the pulse trains, we rely on an engineered arrangement of birefringent crystals to split a pulse in delayed replica. Trains of $2,4,8,16,32$, and 64 pulses are produced by combining 6 crystals of different pre-defined thicknesses. The shortest inter-pulse delay (the one applicable to the 64-pulse trains) of $180 \mathrm{fs}$ between pulses corresponds to an irradiation at a repetition rate of $\cong 5.6 \mathrm{THz}$. The obtained trains of pulses for all crystal combinations are systematically verified by using a long-scan autocorrelator. For irradiation studies, we focus the beam deep inside silicon samples (intrinsic (100), focusing depth exceeding $300 \mu \mathrm{m}$ ) with a microscope objective of $0.85 \mathrm{NA}$. Under these focusing conditions, strong single pulses develop a plasma in the pre-focal region that limits the intensity and energy deposition near the focus. This prevents the occurrence of permanent modifciations $[3,4]$. With the THz-repetition-rate train of weaker pulses, one can accumulate carrier at the focus. The duration of the generated trains exceeding electron-phonon coupling time $(\sim \mathrm{ps})$ but below electron and temperature diffusion times ( ns). Then, we can expect local heating gradually increasing absorption during the train.

To quantify the conditions applied to silicon near focus, we measure the fluence distributions by a Z-scanning microscopy approach similar to the one used in refs. [4]. While the measurement is not possible for the highest NA, we make this analysis for a beam focused with $\mathrm{NA}=0.45$. Then, we estimate from the measurements the absorbed energy density by simple derivation of energy with respect to the position on the optical axis. We also systematically confirm the enhanced conditions in matter by damage test studies for different energy levels, number of pulses in the trains and separation between pulses.

\section{Results and Discussions}

The validity of the THz burst mode of irradiation to enhance the conditions in semiconductors is demonstrated by comparisons on the level of spatio-temporal light energy localization measured under various THz-burst irradiation 
conditions. As shown in figure 1a, measurements reveal for given incoming energy that it exist an optimum number

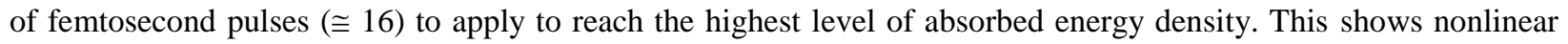
delocalization the beam [8] persist with train of pulses and the new control parameters are used for appropriate excitation build-up and limited retro-reaction.

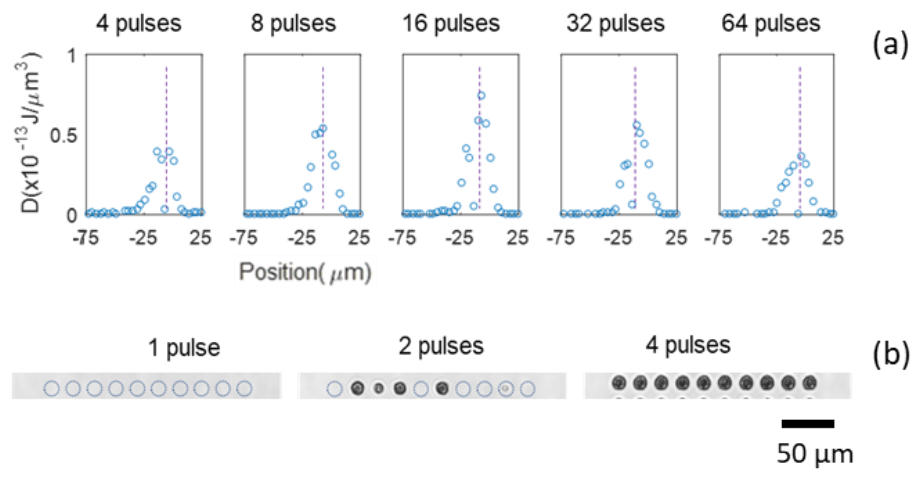

Fig.1. Accumulating energy density with pulse trains. (a) Estimated absorbed energy densities (peak) along the propagation distance (geometrical focus at zero-position) with trains of femtosecond pulses $(5.6 \mathrm{THz})$ focused with $\mathrm{NA}=0.45$ (b) Top-view infrared images of modifications produced with trains of 4-ps pulses at focused $(\mathrm{NA}=0.85)$ $300 \mu \mathrm{m}$ below the surface. All irradiation energies are the same $(2.1 \mu \mathrm{J} /$ train) repeated 10 times for direct comparisons of damage probabilities.

While this level of improvement does not allow to reach the threshold for permanence modification at NA $=0.85$, the benefit of burst mode to limit delocalization of the beam is supported by a range of experiments demonstrating improved 3D writing performances with pulse duration of $\approx 5-7 \mathrm{ps}$. As shown in figure $1 \mathrm{~b}$, while single pulses of 2.1 $\mu \mathrm{J}$ does not allow to modify $\mathrm{Si}$, splitting the energy between 2 pulses, makes possible to cross the threshold for modification despite non-reproducible modification (damage probability $\sim 40 \%$ ). Additional splitting to deliver the same energy with a train of 4 pulses lead to highly repeatable features indirectly showing increased absorbed energy densities in $\mathrm{Si}$ with the number of pulses in the trains.

The THz repetition rate is so high in the tested pulse trains that an important question may arise on the difference on the material response between trains and stretched pulses. While we could not make systematic comparisons to study in details this question, there are already a striking difference revealed with this study. It is the absence of modification with $\mathrm{THz}$ trains of 64-pulses of $200 \mathrm{fs}$ corresponding to an apparent duration of interaction of about $12 \mathrm{ps}$ while single Gaussian pulses of duration exceeding $7 \mathrm{ps}$ can readily produce permanent modifications. This is a direct evidence that the detailed temporal characteristics of irradiation play a major role in these situations. This reveals that the detailed temporal characteristics of irradiation should not be overlooked for the development of ultrafast 3D laser writing technologies applicable to narrow bandgap materials.

\section{Acknowledgements}

This research has received funding from the European Research Council (ERC) under the European Union's Horizon 2020 research and innovation program (Grant Agreement No. 724480).

\section{References}

[1] R. R.. Gattass and E. Mazur "Femtosecond laser micromachining in transparent materials". Nat. Photonics 2, 219-225 (2008).

[2] K. Sugioka and Y. Cheng "Ultrafast lasers-reliable tools for advanced materials processing". Light: Science and Applications 3, (2014)

[3] A. Mouskeftaras, et al. "Self-limited underdense microplasmas in bulk silicon induced by ultrashort laser pulses". Appl. Phys. Lett. 105, 191103 (2014).

[4] M. Chanal, et al. "Crossing the threshold of ultrafast laser writing in bulk silicon". Nat. Commun. 8, 773 (2017).

[5] V.Y. Fedorov et al. "Accessing Extreme Spatiotemporal Localization of High-Power Laser Radiation through Transformation Optics and Scalar Wave Equations. Phys. Rev. Lett. 117, 043902 (2016).

[6] C. Kerse, et al. "Ablation-cooled material removal with ultrafast bursts of pulses". Nature 537, 84-88 (2016).

[7] A. Mouskeftaras, et al. "Direct measurement of ambipolar diffusion in bulk silicon by ultrafast infrared imaging of laser-induced microplasmas". Appl. Phys. Lett. 108, 041107 (2016).

[8] M. Chambonneau, et al. "Competing nonlinear delocalization of light for laser inscription inside silicon with a 2- $\mu \mathrm{m}$ picosecond laser". Phys. Rev. Appl. 12, 024009 (2019). 